\title{
Motion Planning for Active Cannulas
}

\author{
Lisa A. Lyons, Robert J. Webster III, and Ron Alterovitz
}

\begin{abstract}
An active cannula is a medical device composed of thin, pre-curved, telescoping tubes that may enable many new surgical procedures. Planning optimal motions for these devices is challenging due to their kinematics, which involve both beam mechanics and space curves. In this paper, we propose an optimization-based motion planning algorithm that computes actions to guide the device to a target point while avoiding obstacles in the environment. The planner uses a simplified active cannula kinematic model that neglects beam mechanics, and focuses on planning for the (piecewise circular) space curves. The method is intended for use in image-guided procedures where the target and obstacles can be segmented from preprocedure images. Given the target location, the start position and orientation, and a geometric representation of obstacles, the algorithm computes the insertion length and orientation angle for each tube of the active cannula such that the device follows a collision-free path to the target. We formulate the planning problem as a constrained nonlinear optimization problem and use a penalty method to convert this formulation into a sequence of more easily solvable unconstrained optimization problems. Simulations demonstrate optimal paths for a 3-tube active cannula with spherical obstacles. The algorithm typically computes plans in less than 1 minute on a standard PC.
\end{abstract}

\section{INTRODUCTION}

An active cannula is a continuously flexible ("continuum") robot composed of thin, pre-curved, telescoping tubes [32]. They can achieve curved, winding paths to reach distant targets in constrained spaces, as shown in Fig. 1, making them well suited for application in minimally invasive surgery. In an active cannula, bending actuation arises as tubes elastically interact when they slide and rotate within one another. Since active cannulas do not use mechanisms outside the backbone like tendon wires or pneumatic chambers for actuation, they can be very thin without sacrificing dexterity, as shown in Fig. 2.

Planning tube configurations (translations and rotations applied at tube bases) that correspond to desired spatial tip coordinates or shaft curves is not intuitive for human beings, motivating the need for efficient planning algorithms. Active cannulas are actuated by grasping tubes at their bases using manual [32] or robotic [28] actuation units that can apply differential rotations and/or extentions telescopically. Motions applied at the tube bases are transmitted along tube shafts and result in the pre-shaped tube sections causing one another to bend. The net result is a thin, hollow "tentaclelike" device through which a variety of tools, liquids, or

L. A. Lyons and R. Alterovitz are with the Department of Computer Science, University of North Carolina at Chapel Hill, USA llyons@email.unc.edu, ron@cs.unc.edu

R. J. Webster is with the Department of Mechanical Engineering, Vanderbilt University, Nashville, TN, USA robert.webster@vanderbilt.edu

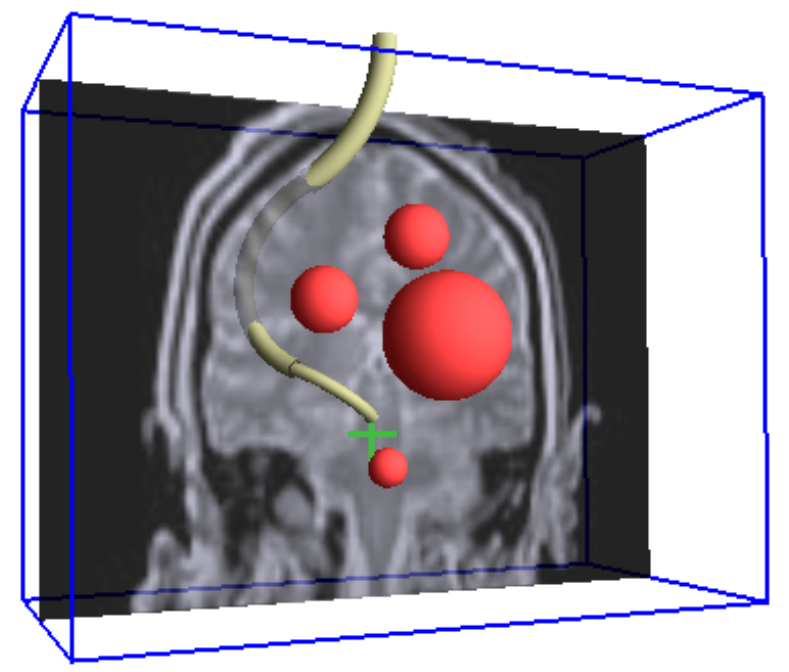

Fig. 1. Illustration of the clinical potential of active cannulas for accessing deep brain structures for biopsy or electrode implantation [18] while avoiding critical structures in an MRI scan [26]. Our motion planner computes a path for an active cannula composed of 3 concentric tubes to reach a target (green cross) while avoiding obstacles (red spheres).

forms of energy may pass and be aimed or manipulated in constrained spaces.

Provided that appropriate motion planning and control algorithms can be developed, active cannulas appear poised to enable minimally invasive access to the lung via the throat [31], [32], the heart [25], the brain for biopsy or electrode implantation [18], and the fetus within the womb [12], and to aid in transnasal skull base and transgastric surgery [14], [16], [30]. These medical procedures require planning the motions for active cannulas through constrained spaces to reach clinical targets.

We propose an optimization-based motion planning algorithm that computes actions necessary to guide the device to a target point while avoiding obstacles in the environment. Obstacles represent tissues that should not be contacted, penetrated, or damaged by the cannula, such as nerves, blood vessels, bones, critical brain structures, or other delicate tissues defined by medical doctors. The planning method is intended for use in image-guided procedures where the target and obstacles can be segmented from pre-procedure images such as Magnetic Resonance Imaging (MRI), Computed Tomography (CT), or Ultrasound. To our knowledge, this is the first algorithm to plan collision-free motions for active cannulas in environments with obstacles.

Our planner determines values for the two input degrees 


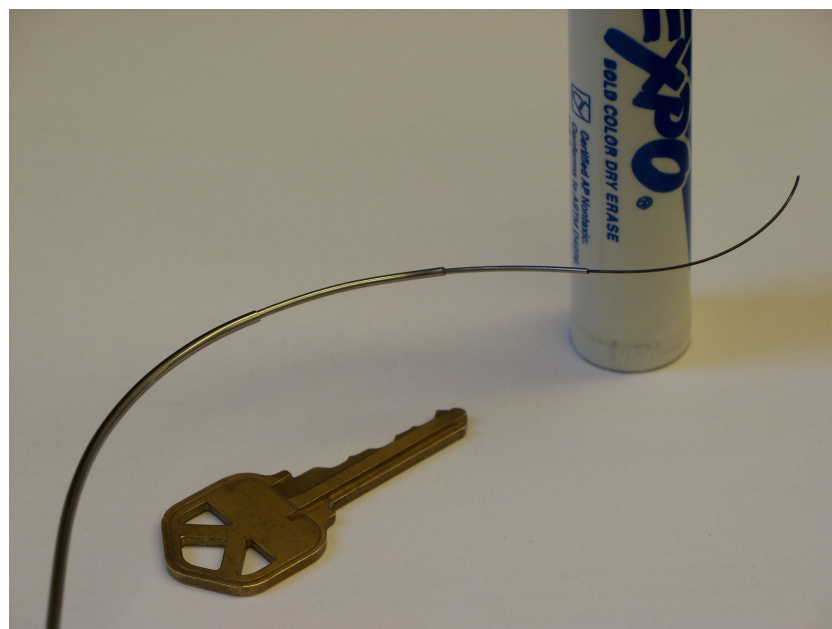

Fig. 2. An active cannula with 4 concentric tubes. Each concentric tube is pre-shaped with a different radius of curvature, with the thinner, inner tubes having higher curvature.

of freedom for each tube in an active cannula: insertion arc length, and axial rotation angle. The planner uses a penalty method and gradient descent to compute locally optimal values for these variables that deliver the cannula to the target while avoiding obstacles.

\section{Related Work}

Prior work has applied optimization-based motion planning to minimally invasive medical procedures, particularly needle insertion. Inserting a needle into soft tissue causes the surrounding tissue to deform. Optimization-based motion planning has been used to compute the insertion location and insertion distance for rigid symmetric-tip needles to compensate for tissue deformations in 2D [3] and in 3D [9]. It has also been applied to flexible, bevel-tip steerable needles that are inserted into deformable soft tissue to reach targets inaccessible to traditional, stiff needles [2].

These planners, using optimization-based motion planning, have focused on computing an optimal configuration for the robot before beginning the motion. The optimization computes the minimum of an objective function surface in configuration space. This builds on the idea behind potential fields, which have been studied extensively for robotic navigation. Potential fields provide a virtual field that robots can follow to a goal while avoiding obstacles [7]. Khatib proposed a model where a goal is represented as an attractor, obstacles are represented as repulsers, and the overall field is determined by the superposition of these fields [17]. Potential fields are used as vector fields that provide the desired velocity vector for the robot at any location in space. Numerous extensions to this approach have been proposed, including methods to prevent the robot from getting trapped at a local minimum [8] and methods to deal with moving obstacles [17], [19]. In this paper, we focus on computing an optimal configuration for an active cannula rather than a vector field.

Active cannulas with a sufficient number of tubes are a type of redundant manipulator. Recent work has explored probabilistic planning algorithms for holonomic redundant manipulators [6], [27]. However, we are not aware of prior work that has considered planning subject to the kinematic constraints of active cannulas.

In terms of mechanical design, active cannulas can be considered a generalization of catheter and needle steering strategies. Base-actuation of a thin flexible device with a circular or annular profile has been widely applied to steer surgical instruments inside the human body. The pervasive catheter is the primary (and often only) means of accessing the human circulatory system minimally invasively. Catheters are directed through a network of blood vessels by "aiming" (through axial rotation) a pre-curved tip down the desired vessel branch, followed by axial insertion along the vessel. Similar input degrees of freedom enable steerable needles to traverse curved paths in soft tissues. One steerable needle design incorporates a precurved tip that extends from a stiffer outer tube to achieve controllable deflection [20]. Another design harnesses the natural bending forces that arise when a standard bevel tip is pushed through tissue, driving the needle along a curved path [29]. Reorienting the tip using axial rotation enables control of needle trajectory [15].

Since they use multiple tubes, active cannulas can be thought of as a multi-element generalization of the baseactuated steering concept. The key difference between active cannulas and single-element designs like catheters or steerable needles is that an active cannula need not rely completely on tissue reaction forces to steer. Rather, it can change shape using internal reaction moments that tubes apply to one another. Thus, active cannulas are properly considered miniature robotic manipulators, and belong to the continuum robot family due to their continuously flexible backbone [24], [32]. The most complete available model of active cannula shape accounts for tube interaction using beam mechanics. It captures the effects of bending, torsion, and variable pre-curvature in an arbitrary number of tubes [24], yielding the shape of the active cannula as a function of tube base translation positions and axial angles. It is the most descriptive and accurate in a series of models [12], [24], [25], [30], [32] derived in the past few years, and includes all prior models as special cases. The simplest of these special cases assumes perfect bending rigidity of each outer tube with respect to all tubes within it, and perfect torsional rigidity of all tubes [12]. Though less accurate than the general model above, this simplest available model provides a good starting point for initial path planning studies, such as the one we describe in this paper.

Prior work has explored motion planning for a variety of continuum robots used in medical applications. Extensive prior work has explored motion planning for steerable needles, which, like active cannulas, follow curved paths. As described above, optimization-based motion planning has been applied to steerable needles inserted in 2D tissue slices around polygonal obstacles [2]. Other approaches include diffusion-based motion planning to numerically compute a path in 3D stiff tissues [21], screw-based motion planning 


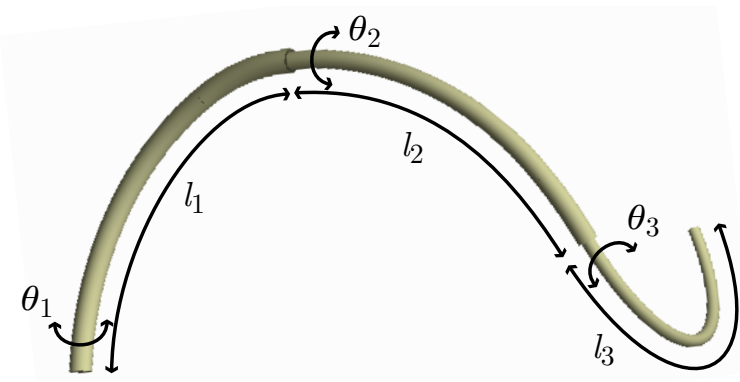

Fig. 3. Configuration $\mathbf{q}=\left(l_{1}, \theta_{1}, l_{2}, \theta_{2}, l_{3}, \theta_{3}\right)$ for an active cannula with $N=3$ concentric tubes.

to compute steerable needle paths in $3 \mathrm{D}$ around spherical obstacles [11], rapidly exploring random trees [33], and planning methods that explicitly consider uncertainty in the needle's motion to maximize the probability that the needle tip successfully reaches the target [1], [4]. The latter method, combined with an imaging and control system, has been successfully integrated with robot hardware [23]. Motion planning methods have also been developed for guiding slightly flexible symmetric-tip needles to targets by translating and orienting the needle base by modeling the deformation of the needle and surrounding tissue [10], [13]. Though the methods described above provide useful intuition about motion planning for continuum robots, they cannot be applied directly to active cannulas due to fundamental differences in the physical mechanisms and their motions.

\section{Problem Formulation}

\section{A. Modeling Assumptions}

Consider an active cannula composed of $N$ tubes. The tubes are numbered in order of decreasing diameter. Each tube is pre-shaped with a constant radius of curvature $r_{i}$, $i=1, \ldots, N$. As discussed in Section II, we follow the simplified kinematic model of [12]. The assumptions of the simplified model are that tubes are torsionally rigid, and that the stiffness of each outer tube dominates all those within it. This implies that each tube deploys along a circular path defined by its radius of curvature, and that its shape is independent of the motions of tubes within it. See [32] for a discussion on the implications of these assumptions, and a comparison of various models to experimental data.

Each tube has two degrees of freedom; it may be extended or retracted from the previous tube and axially rotated, reorienting the direction of deployment, as shown in Fig. 3 . We define a cannula configuration as the $2 N$ dimensional vector $\mathbf{q}=\left(l_{i}, \theta_{i}: i=1, \ldots, N\right)$ where $l_{i}$ is the length of the $i$ 'th tube and $\theta_{i}$ is the rotation of the $i$ 'th tube. We assume that the active cannula is inserted from a start point $\mathbf{x}_{\text {start }}$ and is oriented along the vector $\mathbf{v}_{\text {start }}$.

We define the function $f(\mathbf{q}): \mathbb{R}^{2 N} \mapsto \mathbb{R}^{3}$ as returning the tip position in $3 \mathrm{D}$ space given the active cannula configuration q. We define the transformation $f(\mathbf{q})$ by multiplying a concatenated sequence of translation and rotation matrices that, when translated by the start position, yields the tip position for the active cannula. This function is given by (3) in [28].

We also define $h(\mathbf{q}, s): \mathbb{R}^{6} \times \mathbb{R} \mapsto \mathbb{R}^{3}$ as the function for the curve of the cannula in the configuration defined by $\mathbf{q}$. The curve is parameterized by $s$ in the range $[0,1]$, such that $h(\mathbf{q}, 0)=\mathbf{x}_{\text {start }}$ and $h(\mathbf{q}, 1)=f(\mathbf{q})$. The function $h$ is computed similarly to $f$ using (3) in [28] computed to the arclength defined by $s$ (that is, including only the leftmost several transformations, which define the circular arcs nearest the base of the cannula). Note that in general $s$ will not define a point at the beginning or end of a circular arc. Thus the last (rightmost) transformation uses an appropriate arc length defined by how far along the last circular arc $s$ lies.

Obstacles are defined in a set $O$ of size $M, O=$ $\left\{O_{1}, O_{2}, \ldots, O_{M}\right\}$. Obstacles can be defined using any geometric representation, as long as it is possible to compute $d\left(\mathbf{x}, O_{i}\right)$, the Euclidean distance between a point in space $\mathbf{x}$ and the obstacle $O_{i}$. In our current implementation, we use spherical obstacles.

\section{B. Planning Problem Formulation}

We define the target by the coordinate $\mathbf{x}_{\text {target }}$ in 3D space. We assume the start location $\mathbf{x}_{\text {start }}$ and initial orientation $\mathbf{v}_{\text {start }}$ are given and fixed implying that the physician has a single opening through which the cannula may be inserted based on the constraints of the procedure. In future work, we will consider treating $\mathbf{x}_{\text {start }}$ and $\mathbf{v}_{\text {start }}$ as variables for cases in which the physician may select the insertion pose of the active cannula as part of the procedure.

Given the active cannula properties, the start pose, the target coordinate, and the obstacles, we formulate the motion planning problem as a nonlinear, constrained optimization problem. First, we require that the active cannula tip reach the target, which we represent as the constraint:

$$
\left\|f(\mathbf{q})-\mathbf{x}_{\text {target }}\right\|=0 \text {. }
$$

Second, we prefer solutions that avoid obstacles. We quantify this using a potential field [17] by integrating the sum of the obstacle potential field values along the path of the active cannula:

$$
c(\mathbf{q})=\sum_{i=1}^{M} \int_{s=0}^{1} \frac{1}{d\left(h(\mathbf{q}, s), O_{i}\right)^{2}} d s .
$$

Finally, we require that the active cannula configuration be valid, which we represent with the constraints $l_{i} \geq 0, \theta_{i} \geq 0$, and $\theta_{i}<2 \pi, i=1, \ldots, N$.

The motion planning problem may now be formulated as a nonlinear, constrained optimization problem:

$$
\begin{array}{ll}
\mathbf{q}^{*}=\underset{\mathbf{q}}{\operatorname{argmin}} c(\mathbf{q}) & \\
\text { Subject to: } & \\
\left\|f(\mathbf{q})-\mathbf{x}_{\text {target }}\right\|=0 & \\
l_{i} \geq 0 & i=1, \ldots, N \\
\theta_{i} \geq 0 & i=1, \ldots, N \\
\theta_{i}<2 \pi & i=1, \ldots, N
\end{array}
$$

where $\mathbf{q}^{*}$ is the optimal active cannula configuration. Because we assume that the stiffness of each outer tube dominates 

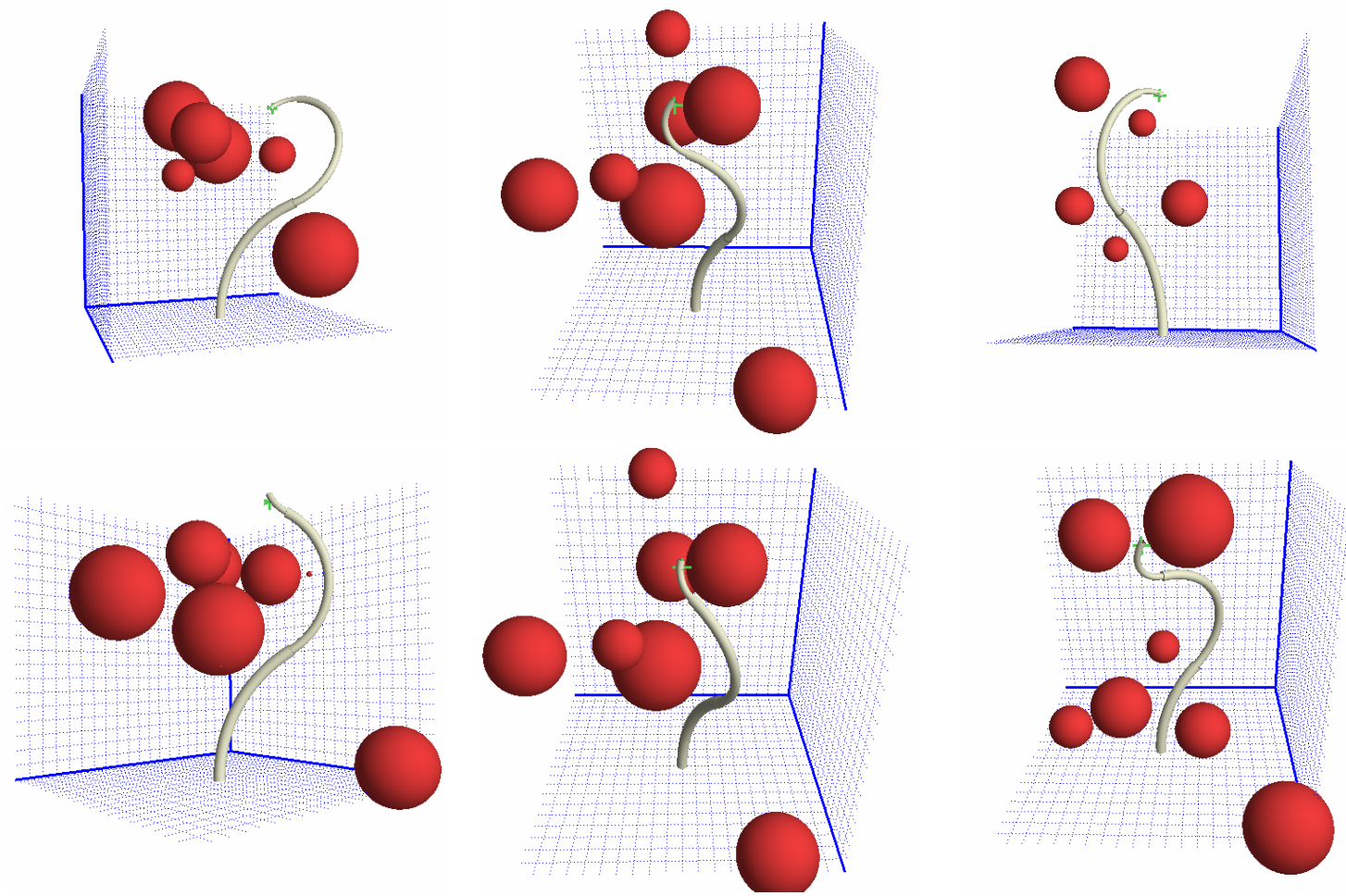

Fig. 4. Active cannula plans computed by the method for test cases with randomly placed spherical obstacles.

those within it (implying that it will deploy along its own radius of curvature and stay there as other tubes deploy within it), we can achieve this optimal configuration by first axially rotating the outer tube to $\theta_{1}$, then deploying it to a length $l_{1}$, and then repeating for tubes $i=2, \ldots, N$ in increasing order.

\section{Motion Planning Algorithm}

Using the optimization-based formulation in (2), solving the motion planning problem requires solving the constrained, nonlinear optimization problem. We solve for a locally optimal solution $\mathbf{q}^{*}$ using a penalty method. Penalty methods solve a constrained optimization problem by converting it to a series of unconstrained optimization problems [5]. Given the constrained optimization problem $\min f(\mathbf{x})$ subject to $g(\mathbf{x}) \leq 0$, we can write the unconstrained problem $\min (f(\mathbf{x})+\mu \max \{0, g(\mathbf{x})\})$ for some large $\mu>0$. Penalty methods generate a series of unconstrained optimization problems as $\mu \rightarrow \infty$. Each unconstrained optimization problem can be solved using Gradient Descent or variants of Newton's Method. For convex nonlinear problems, the method will generate points that converge arbitrarily close to the global optimal solution [5]. For nonconvex problems, the method can only converge to a local optimal solution.

Applying the penalty method to the active cannula motion planning problem, we move the non-trivial constraint $\left\|f(\mathbf{q})-\mathbf{x}_{\text {target }}\right\|=0$ to the objective function and define a new optimization problem.

$$
\begin{array}{cc}
\mathbf{q}^{*}=\underset{\mathbf{q}}{\operatorname{argmin}}\left(c(\mathbf{q})+\mu\left\|f(\mathbf{q})-\mathbf{x}_{\text {target }}\right\|\right) \\
\text { Subject to: } & \\
l_{i} \geq 0 & i=1, \ldots, N \\
\theta_{i} \geq 0 & i=1, \ldots, N \\
\theta_{i}<2 \pi & i=1, \ldots, N
\end{array}
$$

The remaining constraints are limit constraints that can easily be handled by unconstrained minimization algorithms.

For a given $\mu$, we use the Gradient Descent algorithm [22] to find a locally optimal solution for (3). We compute the gradient numerically. For each gradient direction, we use Armijo's Rule [5] for line search with an initial movement distance of $\epsilon=.0001$.

We initially set $\mu=10000$ and increase $\mu$ by a factor of $\beta=10$ at each penalty method iteration. To ensure that the constraint $\left\|f(\mathbf{q})-\mathbf{x}_{\text {target }}\right\|=0$ is satisfied, the penalty method is executed until the tip is within some small Euclidean distance $\delta$ of the target. Decreasing $\delta$ results in more accurate convergence to the target but requires more computation time.

Computing $c(\mathbf{q})$ in (1) requires numerically approximating an integral over the active cannula path. We approximate this integral by considering a finite set of points spaced $a$ apart along the active cannula path and converting the integral to a summation.

Because we compute locally optimal solutions to the optimization problem in (3), the planner may fail to find a quality solution because it is trapped in a local minimum. To address this, we select 10 random, uniformly distributed 
initial configurations of $\mathbf{q}$ and execute the local optimization on each initial configuration. The initial configurations were chosen using the following criteria:

$$
\begin{aligned}
& 0 \leq \theta_{i} \leq 2 \pi, \quad i=1,2,3 \\
& 0 \leq l_{i} \leq r_{i}, \quad i=1,2,3
\end{aligned}
$$

Although we automatically select the optimal solution based on the lowest value of $c(\mathbf{u})$, physicians or engineers may also choose among the solutions found to optimize other criteria, such as shortest tube deployment lengths, minimum torsion, more rapidly healing tissues, etc.

\section{Results}

We illustrate the results of the planner in Fig. 1 and Fig. 4 for active cannulas with $N=3$ tubes. All visualizations were done using OpenGL. The results show that the planner is able to find paths around spherical obstacles to targets specified in 3D space. Each of these plans was computed in under 1 minute on a $2.00 \mathrm{GHz}$ Intel@ Core $^{\mathrm{TM}} 2$ Duo PC with 2 GB RAM.

To evaluate the performance of the method, we ran experiments in five test environments, shown in Fig. 5. For each test environment, a different random configuration of six randomly sized obstacles was used in a workspace of dimension $20 \mathrm{~cm} \times 20 \mathrm{~cm} \times 20 \mathrm{~cm}$. In all experiments, we used active cannulas with $N=3$ concentric tubes with radii of curvature of $r_{1}=10 \mathrm{~cm}, r_{2}=5 \mathrm{~cm}$, and $r_{3}=2 \mathrm{~cm}$.

For these experiments, we set $\delta=0.3 \mathrm{~cm}$ for target constraint satisfaction and set point spacing $a=0.5 \mathrm{~cm}$ for cost computation along the active cannula path. Since the $\operatorname{cost} c(\mathbf{u})$ is computed at every iteration of the optimization and is one of the most expensive routines, it is crucial to set $a$ to attain an accurate but relatively inexpensive parameterization. For numerical stability, $\mu$ was only allowed to increase by a maximum factor of 1000 from its initial value.

For each test environment, we randomly selected 100 target locations using a uniform distribution inside the boxes shown in Fig. 5. Sample target coordinates inside obstacles were removed from consideration. For each target, we solve the optimization for each of the ten randomly chosen initial conditions to determine if a solution can be found using any of them.

We achieved a $100 \%$ success rate for reaching the goal and not colliding with any obstacles. Table I shows the average, standard deviation, and median time per trial for each of the five test environments. All tests were run on a $2.00 \mathrm{GHz}$ Intel@Core ${ }^{\mathrm{TM}_{2}}$ Duo PC with 2 GB RAM. The large standard deviations are caused by the variation in difficulty of the task for random target positions. For instance, a target that is obstructed by two obstacles requires more planning time than a target with no obstructions. Test environment (d) was the most difficult for the planner because it had many obstacles surrounding the target region.

\section{Conclusion}

In this paper, we propose an optimization-based motion planning algorithm for active cannulas that computes actions



(a)

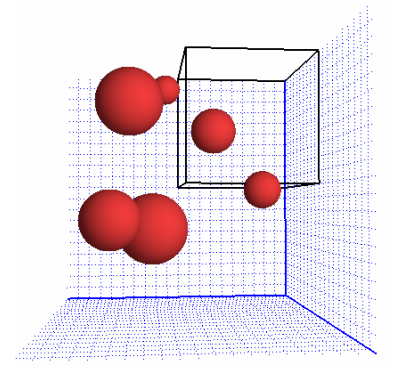

(b)

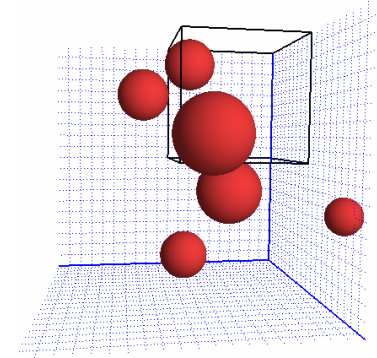

(d)

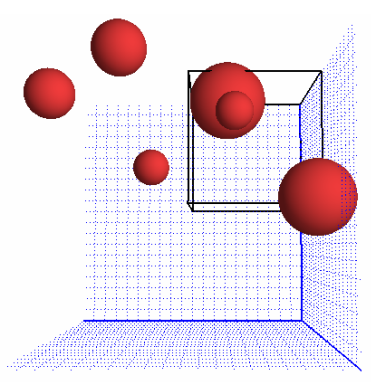

(c)

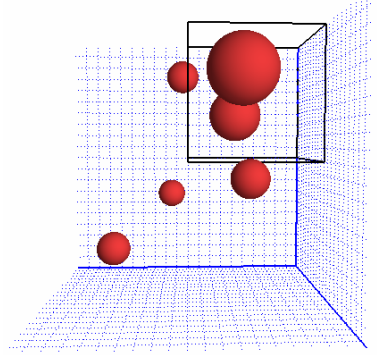

(e)
Fig. 5. Five test environments, each containing 6 obstacles. Targets were selected randomly using a uniform distribution inside the black boxes; samples inside an obstacle were removed.

to guide the device to a target point while avoiding obstacles in the environment. Given the target location, the start position and orientation, and a geometric representation of the obstacles, the algorithm computes the insertion length and orientation angle for each tube of the active cannula such that the device follows a collision-free path to the target. We formulated the planning problem as a constrained nonlinear optimization problem and used a penalty method to convert this formulation into a sequence of more easily solvable unconstrained optimization problems. We evaluated the method for 3-tube active cannulas operating in environments with spherical obstacles. For five test environments composed of randomly located spherical obstacles, the method found a solution $100 \%$ of the time. The algorithm typically computes plans in less than 1 minute on a standard PC.

In future work, we plan to take steps to bring motion planning for active cannulas closer to clinical application. The planning algorithm is intended for use in image-guided procedures where the target and obstacles can be segmented from pre-procedure images. We plan to extend the implementation to properly handle obstacles defined by tetrahedral meshes based on segmented tissue structures. We also plan 
TABLE I

COMPUTATION TIMES FOR 100 tRIALS IN FIVE RANDOM OBSTACLE ENVIRONMENTS. All tests were run on a $2.00 \mathrm{GHz}$ Intel $\left(\mathrm{CoRE}^{\mathrm{TM}} 2\right.$ Duo PC with $2 \mathrm{~GB}$ RAM.

\begin{tabular}{|l|r|r|r|}
\hline $\begin{array}{l}\text { Test } \\
\text { Environment }\end{array}$ & $\begin{array}{r}\text { Median Time } \\
(\mathrm{s})\end{array}$ & $\begin{array}{r}\text { Mean time } \\
(\mathrm{s})\end{array}$ & $\begin{array}{r}\sigma \\
(\mathrm{s})\end{array}$ \\
\hline (a) & 11.79 & 15.81 & 12.46 \\
\hline (b) & 13.05 & 21.01 & 20.99 \\
\hline (c) & 6.33 & 11.03 & 17.33 \\
\hline (d) & 20.48 & 295.09 & 25.51 \\
\hline (e) & 0.34 & 1.38 & 4.62 \\
\hline
\end{tabular}

to improve the accuracy of our active cannula model by using the the most recent models which account for bending, torsion, and arbitrary precurvature functions that are not required to be circular [24]. We also plan to develop new motion planning algorithms for active cannulas capable of finding globally optimal solutions with guarantees on success rate and to compute plans that consider uncertainty in active cannula motion and obstacle locations. Finally, we plan to integrate motion planning for active cannulas with robot control hardware to automatically guide these devices to targets while avoiding obstacles.

\section{AcKNOWLEDGEMENT}

The authors thank Caleb Rucker for the photo in Fig. 2.

\section{REFERENCES}

[1] R. Alterovitz, M. Branicky, and K. Goldberg, "Motion planning under uncertainty for image-guided medical needle steering," Int. J. Robotics Research, vol. 27, no. 11-12, pp. 1361-1374, Nov. 2008.

[2] R. Alterovitz, K. Goldberg, and A. M. Okamura, "Planning for steerable bevel-tip needle insertion through 2D soft tissue with obstacles," in Proc. IEEE Int. Conf. Robotics and Automation (ICRA), Apr. 2005, pp. 1652-1657.

[3] R. Alterovitz, J. Pouliot, R. Taschereau, I.-C. Hsu, and K. Goldberg, "Sensorless planning for medical needle insertion procedures," in Proc. IEEE/RSJ Int. Conf. on Intelligent Robots and Systems (IROS), Oct. 2003, pp. 3337-3343.

[4] R. Alterovitz, T. Siméon, and K. Goldberg, "The Stochastic Motion Roadmap: A sampling framework for planning with Markov motion uncertainty," in Robotics: Science and Systems III (Proc. RSS 2007), W. Burgard, O. Brock, and C. Stachniss, Eds. Cambridge, MA: MIT Press, 2008, pp. 246-253.

[5] M. S. Bazaraa, H. D. Sherali, and C. M. Shetty, Nonlinear Programming: Theory and Algorithms, 2nd ed. New York, NY: John Wiley \& Sons, Inc., 1993.

[6] D. Bertram, J. Kuffner, R. Dillmann, and T. Asfour, "An integrated approach to inverse kinematics and path planning for redundant manipulators," in Proc. IEEE Int. Conf. Robotics and Automation (ICRA), May 2006, pp. 1874-1879.

[7] H. Choset, K. M. Lynch, S. Hutchinson, G. Kantor, W. Burgard, L. E. Kavraki, and S. Thrun, Principles of Robot Motion: Theory, Algorithms, and Implementations. MIT Press, 2005.

[8] C. I. Connolly, J. B. Burns, and R. Weiss, "Path planning using Laplace's equation," in Proc. IEEE Int. Conf. Robotics and Automation (ICRA), May 1990, pp. 2102-2106.

[9] E. Dehghan and S. E. Salcudean, "Needle insertion point and orientation optimization in non-linear tissue with application to brachytherapy," in Proc. IEEE Int. Conf. Robotics and Automation (ICRA), Apr. 2007, pp. 2267-2272.

[10] S. P. DiMaio and S. E. Salcudean, "Needle steering and model-based trajectory planning," in Medical Image Computing and Comput. Asst. Intervention (MICCAI), 2003, pp. 33-40.

[11] V. Duindam, R. Alterovitz, S. Sastry, and K. Goldberg, "Screw-based motion planning for bevel-tip flexible needles in $3 \mathrm{~d}$ environments with obstacles," in Proc. IEEE Int. Conf. Robotics and Automation (ICRA), May 2008, pp. 2483-2488.
[12] J. Furusho, T. Katsuragi, T. Kikuchi, T. Suzuki, H. Tanaka, Y. Chiba, and H. Horio, "Curved multi-tube systems for fetal blood sampling and treatments of organs like brain and breast," J. Comput. Assist. Radiol. Surg., vol. 1, pp. 223-226, 2006.

[13] D. Glozman and M. Shoham, "Image-guided robotic flexible needle steering," IEEE Trans. Robotics, vol. 23, no. 3, pp. 459-467, June 2007.

[14] H. M. Hegazy, R. L. Carrau, C. H. Snyderman, A. Kassam, and J. Zweig, "Transnasal endoscopic repair of cerebrospinal fluid rhinorrhea: A meta-analysis," Laryngoscope, vol. 110, pp. 1166-1172, 2000.

[15] V. Kallem and N. J. Cowan, "Image guidance of flexible tip-steerable needles," IEEE Trans. Robotics, vol. 25, no. 1, pp. 191-196, 2009.

[16] A. B. Kassam, C. Snyderman, P. Gardner, R. Carrau, and R. Spiro, "The expanded endonasal approach: A fully endoscopic transnasal approach and resection of the odontoid process: Technical case report," Operative Neurosurg., vol. 57, pp. 213-214, 2005.

[17] O. Khatib, "Real-time obstacle avoidance for manipulators and mobile robots," Int. J. Robotics Research, vol. 5, no. 1, pp. 90-98, 1986.

[18] A. J. Martin, W. A. Hall, C. Roark, P. A. Starr, P. S. Larson, and C. L. Truwit, "Minimally invasive precision brain access using prospective stereotaxy and a trajectory guide." J Magn Reson Imaging, vol. 27, no. 4, pp. 737-743, Apr 2008.

[19] W. S. Newman and N. Hogan, "High speed robot control and obstacle avoidance using dynamic potential functions," in Proc. IEEE Int. Conf. Robotics and Automation (ICRA), 1987, pp. 14-24.

[20] S. Okazawa, R. Ebrahimi, J. Chuang, S. E. Salcudean, and R. Rohling, "Hand-held steerable needle device," IEEE/ASME Trans. Mechatronics, vol. 10, no. 3, pp. 285-296, June 2005.

[21] W. Park, J. S. Kim, Y. Zhou, N. J. Cowan, A. M. Okamura, and G. S. Chirikjian, "Diffusion-based motion planning for a nonholonomic flexible needle model," in Proc. IEEE Int. Conf. Robotics and Automation (ICRA), Apr. 2005, pp. 4611-4616.

[22] W. H. Press, B. P. Flannery, S. A. Teukolsky, and W. T. Vetterling, Numerical Recipes in C: The Art of Scientific Computing, 2nd ed. Cambridge University Press, 1992.

[23] K. B. Reed, V. Kallem, R. Alterovitz, K. Goldberg, A. M. Okamura, and N. J. Cowan, "Integrated planning and image-guided control for planar needle steering," in Proc. IEEE/RAS-EMBS Int. Conf. on Biomedical Robotics and Biomechatronics (BioRob), Oct. 2008, pp. 819-824.

[24] D. C. Rucker and R. J. Webster III, "Parsimonious evaluation of concentric-tube continuum robot equilibrium conformation," IEEE Transactions on Biomedical Engineering Letters, 2009, in press.

[25] P. Sears and P. Dupont, "A steerable needle technology using curved concentric tubes," in Proc. IEEE/RSJ Int. Conf. on Intelligent Robots and Systems (IROS), Oct. 2006, pp. 2850-2856.

[26] The National Library of Medicine, "Visible human project," Available: http://www.nlm.nih.gov/research/visible/visible\%5Fhuman.html, 2003.

[27] J. M. Vandeweghe, D. Ferguson, and S. Srinivasa, "Randomized path planning for redundant manipulators without inverse kinematics," in IEEE-RAS Int. Conf. on Humanoid Robots, November 2007.

[28] R. J. Webster III, J. P. Swenson, J. M. Romano, and N. J. Cowan, "Closed-form differential kinematics for concentric-tube continuum robots with application to visual servoing," in Experimental Robotics: The 11th International Symposium, ser. STAR, O. Khatib et al., Eds. Berlin, Germany: Springer-Verlag, 2009, vol. 54, pp. 485-494.

[29] R. J. Webster III, J. S. Kim, N. J. Cowan, G. S. Chirikjian, and A. M. Okamura, "Nonholonomic modeling of needle steering," Int. J. Robotics Research, vol. 25, no. 5-6, pp. 509-525, May 2006.

[30] R. J. Webster III, A. M. Okamura, and N. J. Cowan, "Toward active cannulas: Miniature snake-like surgical robots," in Proc. IEEE/RSJ Int. Conf. on Intelligent Robots and Systems (IROS), 2006, pp. 2857-2863.

[31] _ - "Kinematics and calibration of active cannulas," in Proc. IEEE Int. Conf. Robotics and Automation (ICRA), 2008, pp. 3888-3895.

[32] R. J. Webster III, J. M. Romano, and N. J. Cowan, "Mechanics of precurved-tube continuum robots," IEEE Trans. Robotics, vol. 25, no. 1, pp. 67-78, Feb. 2009.

[33] J. Xu, V. Duindam, R. Alterovitz, and K. Goldberg, "Motion planning for steerable needles in $3 \mathrm{~d}$ environments with obstacles using rapidlyexploring random trees and backchaining," in Proc. IEEE Int. Conf. Automation Science and Engineering (CASE), Aug. 2008, pp. 41-46. 